\title{
Randomisation in a clinical trial: is it feasible in the Ebola crisis? If it is, then which method is suitable?
}

\author{
Jesca Mercy Batidzirai* \\ aSchool of Mathematics, Statistics and Computer Science, University of KwaZulu-Natal, Pietermaritzburg, South Africa \\ *Email: Batidzirai@ukzn.ac.za
}

Randomisation is an integral component of any sensible clinical trial. Randomisation is the only way we can be sure that the patients have been allocated into the treatment arms with as minimum bias as possible, and that the treatment arms are similar before the start of the trial. The randomisation schemes used to allocate patients into the treatment arms play a role in achieving this goal. This study is motivated by the outbreak of Ebola in West Africa. Scientists claim to have developed some cure for the deadly disease. The question is: 'Ethically, can the trials be non-randomised due to the crisis? If the trials are to be randomised, which randomisation method is suitable?' An overview of the randomisation methods was done and recommended stratified randomisation of Ebola patients between any new treatment and the conventional care is provided. Each of the involved countries or centres was treated as the strata and, within each stratum, simple randomisation was then performed.

Keywords: Ebola, placebo, prognostic factors, randomisation, statistical power

The conduct of randomised controlled trials (RCT's) has become a controversial issue in the Ebola trials since its outbreak in March 2014. In Phase III of any RCT, the efficacy of two different treatment interventions is compared. These can either be the new treatment against the standard treatment that is already on the market, or the new treatment against a placebo (which, in line with The Declaration of the Helsinki Ethical Principles, is acceptable where there is no effective standard treatment that already exists). Two groups of patients are used to compare these two treatment effects, namely the treatment group and the control group. The treatment group receives the new treatment, whilst the control group receives either the standard treatment or the placebo. Patients are selected from heterogeneous populations; hence, the two groups may have many differences in their baseline characteristics that may influence the comparison between them. At the beginning of the study, the treatment groups should be fairly equal in their baseline characteristics, so they should produce almost similar results when given the same treatment. Fairly equal groups allow the researcher to conclude that any difference in the results is attributable to treatment differences'.

To try to make the treatment groups as similar as possible and to balance out the covariate factors between them (whether known or unknown), patients are allocated at random to either of the treatment groups so that they receive either of the treatments a process called randomisation, introduced by Fisher ${ }^{2}$. This helps guard against any use of judgement or systematic arrangement that can lead to bias. Randomisation is, therefore, defined as a technique for randomly allocating patients by chance, rather than by choice, into the treatment groups of a clinical trial; and, is mainly done to balance out conscious or unconscious prognostic factors. This achieves balance and reduces bias, respectively.

Friedman et $a^{\beta}$ confirmed that the process of randomisation tends to generate study groups comparable with respect to known and unknown risk factors; removes investigator bias in the allocation of participants; and, guarantees that statistical tests will have valid significance levels.
To appreciate randomisation in clinical trials, Pocock ${ }^{4}$ gives examples where randomisation was not done resulting in the treatment group and the control group differing in more than just the treatment they received. It becomes difficult to conclude that the improvement or reduction in the endpoint for the new treatment is genuinely due to the treatment effect alone. To date, most clinicians have used randomisation to allocate participants in their clinical trials. Each patient will have a non-zero, usually an equal, probability of receiving any of the treatments under study. In carrying out a clinical trial, therefore, the treatment groups are made as homogeneous as possible before the treatment commences. Where possible, blinding can also be used to complement randomisation.

With some organisations calling for Ebola treatment trials to be fast-tracked in West Africa ${ }^{5}$, pressure is put on clinicians and there is a temptation not to follow ethical procedures. A suitable randomisation method for Ebola trials in such a short space of time must be identified.

Adebamowo et al ${ }^{6}$ suggest that 'there could be other appropriate methods to identify drug regimens that improve outcomes over the existing methods of care'. They do not agree that randomisation is the only way to gather reliable evidence about safety and effectiveness of potential Ebola therapies. The Lancet ${ }^{7}$ argued that 'although RCTs provide robust evidence in most circumstances, alternative trial designs need to be considered because of lack of effective treatment options for Ebola; high mortality with the current standard care; and, the paucity of effective health care systems in the affected regions'.

On the other hand, Shaw ${ }^{8}$ pointed out that 'to abandon usual protocols and give all participants a new drug that turns out to be harmful could also weaken trust'. He went on to mention that since the supply of Zmapp and other Ebola treatments are limited, then RCTs are the best way to generate evidence with these small supplies. According to Cox et $\mathrm{al}^{9}$, properly designed RCT's that give reliable answers are critical to identifying urgently 
needed treatments in response to the ongoing Ebola crisis and any future research. With these arguments, it makes sense to scrutinise the available randomisation methods and recommend one for such large trials, as in the case of Ebola.

\section{Methods of randomisation}

Simple randomisation is a method where there are no restrictions imposed on the nature of the allocation sequence. Each patient has an equal chance of being allocated to either of the treatment groups. This method has the advantage of complete unpredictability of the next treatment allocation. Simple randomisation is easy to implement and is also not discoverable, i.e. if anyone uses exactly the same method and same command, there is no guarantee of obtaining the same allocation results. Another advantage of simple randomisation is that it allows patients to arrive sequentially into the trial and still be randomised and receive the treatment. One disadvantage of simple randomisation is that it can produce unbalanced groups in terms of size and baseline characteristics. However, if the sample is large, simple randomisation produces well balanced treatment groups by chance. ${ }^{10}, 11$ The probability theory guarantees that the sizes of both treatment groups will not be completely different in the long run.

Block randomisation helps overcome the problem of imbalances in numbers as well as chronological bias between treatment groups. Blocks with equal numbers of A's and B's are used where the order of treatments within the block are randomly permuted (where for example, $A=$ new treatment; and, $B=$ control). Usually, four or six treatment assignments are randomly ordered as blocks, such that within each block, there will be an equal number of patients allocated to each of the treatment arms. Therefore, it ensures that treatment groups are almost equally distributed in each block. ${ }^{12}$ Though blocking balances out treatment group numbers in the end of each block, it may lead to the allocation being unconcealed (reduced unpredictability). This is because, if the physician discovers the block size, he/she can easily predict to which arm the next participant is going to be allocated. To try to eliminate this, three things can be done, namely, double blinding, using blocks of bigger sizes; or, varying the block size randomly throughout the allocation process.

Stratified Randomisation solves the problem of imbalance in terms of baseline characteristics such as age, gender, disease history, centre, etc. Stratifying is done so that comparison of treatments can be done within relatively homogenous groups at baseline. Participants are partitioned into mutually exclusive subsets defined by initial baseline characteristics, which are thought to influence the outcome. This is achieved by grouping participants with the same characteristics in the same stratum, and then performing separate block randomisations for each stratum (random permuted blocks) or simple randomisation for each stratum. By so doing, we make sure that the sample is representative.

If stratification was used for randomisation, it should be taken into account when analysing the results because not accounting for stratification in the analysis may result in overestimation of the $p$-value for a difference between endpoint rates in treatment groups. Stratification increases statistical power but has a weakness that, if there are too many prognostic factors that are identified, it may result in chance imbalances between the treatment groups. Another drawback of stratified randomisation lies in its need to identify all the participants in the study, so that they can be allocated to their strata. This becomes a problem since participants come into the study successively. Another disadvantage is that the confounding factor used to stratify may not be important to the outcome of the study and some more factors may be identified later to be of more importance.

Minimisation is a covariate adaptive random allocation method which balances the marginal distribution of each prognostic factor, balances several prognostic factors between the two treatment arms, as well as the number of participants throughout the trial. Minimisation is so termed because the unevenness in the distribution of prognostic factors is minimised. Minimisation is a non-random method of allocation, even though the first person is randomly allocated. This method has been recommended by several researchers as a valid alternative randomisation method for clinical trials. Each subsequent participant's treatment allocation is determined so as to even out size and prognostic factors between the treatment arms, depending on the participants who were already allocated to the treatment groups.

\section{Results and conclusions}

Before choosing a randomisation method, the researcher should consider such things as enrolment of participants; size of clinical trial; need of balancing in sample size; and, baseline characteristics. Suresh ${ }^{11}$ acknowledged that 'randomisation ensures against the accidental bias in the experiment and produces comparable groups in all respects except the intervention each group received'. Therefore, the efficacy of any new Ebola treatment has to be tested - just in case it has a higher mortality rate than conventional care, in which case, conventional care should be retained. Furthermore, it shows that randomisation needs to be implemented. RCT's must be conducted regardless of how much pressure is at hand or how serious the disease is, since the efficacy of the treatment still needs to be tested.

In order to make inferences about its effectiveness, the new treatment for the population with a particular disease must be administered to an adequately sized and representative sample of participants ${ }^{4}$. The sample size must be sufficiently large to reveal a true difference between treatment groups. In large clinical research, simple randomisation can be trusted to generate similar numbers of subjects among groups ${ }^{11}$. As the World Health Organization reported, there were 21296 suspected cases and 8429 deaths due to Ebola by 14 January 2015 (WHO, 2015) ${ }^{13}$.

To date, there are no baseline characteristics known to affect the treatment of Ebola. However, since there are several countries involved, there could be a possibility of bias due to the countries and/or centres to which the patients report. These variations could result from differences in climate; availability of proper health care; accessibility to proper health care; etc. In such cases, the countries and/or centres involved can be treated as the strata, and simple randomisation then performed within each of the countries.

In the case of Ebola trials, block randomisation and minimisation are not relevant since, when the sample size is as big as the one at hand, simple randomisation produces equal numbers among the treatment group by chance ${ }^{11}$. Although block randomisation and minimisation methods increase the statistical power, they would first require the identification of all patients in the trial before performing the randomisation. Therefore, simple randomisation within each stratum is the only practical way to approach randomisation for Ebola trials. The main reason for 
performing simple randomisation in each country and/or centre involved in Ebola trials is its practicality as patients enter the trial successively. Therefore, given the evidence presented, it is recommended that stratified randomisation is used, where the countries and/or centres are the strata, and simple randomisation is used within each stratum.

Conflict of interest - Author does not have either a commercial or other association that might pose as a conflict of interest.

\section{References}

1. Beller EM, Gebski V, Keech AC. Randomisation in clinical trials. Med J Aust. 2002;177(10):565-7.

2. Fisher RA. The arrangement of field experiments. J Min Agric Gr Br. 1926;33:700-25.

3. Friedman LM, Furberg CD, DeMets DL. Fundamentals of clinical trials. 3rd ed. New York: Springer Verlag. 1998; p. 61

4. Pocock SJ. Allocation of patients to treatment in clinical trials. Biometrics. 1979;35(1):183-97.

5. Welcome Trust. Ebola treatment trials to be fast-tracked in West Africa. 2014 [cited 2014 Oct 9]. Available from: http://www.wellcome. ac.uk/News/Media-office/Press-releases/2014/WTP057419.htm

6. Adebamowo C, Bah-Sow O, Binka F, et al. Randomised controlled trials for Ebola: practical and ethical issues. The Lancet 2014;384(9952):1423-4.
7. The Lancet. Insisting only on randomized controlled trials for Ebola treatments unethical, impractical, say leading health experts. [cited 2014 Dec 10]. Available from: http://www.sciencedaily.com/ releases/2014/10/141010134326.htm

8. Shaw D, Randomisation is essential in Ebola drug trials. Lancet. 2014 [cited 2014 Dec 9];384(9955):1667. Available from: http://www. thelancet.com/journals/lancet/article/PIIS0140-6736(14)61735-9/ fulltext

9. Cox E, Brorio L Temple R. Evaluating Ebola therapies-the case for RCTs. N Engl J Med.. 2014 [cited 2014 Dec 12]. Available from: http://www. nejm.org/doi/full/10.1056/NEJMp1414145

10. Lachin JM. Properties of simple randomisation in clinical trials. Control Clin Trials. 1988;9(4):312-26.

11. Suresh KP. An overview of randomisation techniques: an unbiased assessment of outcome in clinical research. J Hum Reprod Sci. 2011;4(1):8-11.

12. Efird JT, Bith-Melander P, Kimata C, et al. SAS Algorithm for Blocked Randomisation. 2007 Paper 2582-2007. [cited 2014 Dec 12]. Available from: http://www.lexjansen.com/wuss/2007/CodersCorner/COD Efird_SASAlgorithm.pdf

13. World Health Organization. Ebola response roadmap situation report. 2014 [cited 2015 Jan 15]. Available from: http://apps.who. int/iris/bitstream/10665/148237/2/roadmapsitrep_14Jan2015_eng. pdf?ua $=1$ 